\title{
Determinação da necessidade de adubação nitrogenada de cobertura no feijoeiro irrigado com auxílio do clorofilômetro portátil
}

\author{
Topdressing nitrogen fertilizer requirements determination for irrigated common bean, by leaf \\ portable chlorophyll meter
}

\author{
Morel Pereira Barbosa Filho ${ }^{*}$ Tarcisio Cobucci ${ }^{\mathrm{I}}$ Nand Kumar Fageria ${ }^{\mathrm{I}}$ \\ Patrícia Neves Mendes ${ }^{\text {II }}$
}

\section{RESUMO}

As pesquisas demonstram que as leituras de transmitância obtidas em clorofilômetro portátil podem indicar a época de maior demanda de nitrogênio (N) das plantas e se há ou não a necessidade de sua aplicação em cobertura. Este foi o objetivo do presente trabalho, que avaliou para a cultura do feijoeiro irrigado os seguintes manejos de $\mathrm{N}$ : $\mathrm{M1}$ : $240 \mathrm{~kg} \mathrm{ha}^{-1}$ de $N$ (80kg na semeadura $80 \mathrm{~kg}$ aos 15 dias após emergência (dae) $+80 \mathrm{~kg}$ aos 30 dae), M2: $120 \mathrm{~kg} \mathrm{ha}^{-1}$ (30 de $\mathrm{N}$ na semeadura $+45 \mathrm{~kg}$ aos 15 dae $+45 \mathrm{~kg}$ aos 30 dae); $\mathrm{M3}: 30 \mathrm{~kg}$ $h^{-1}$ de $N$ na semeadura e aplicação em cobertura estabelecida com base no uso do clorofilômetro Minolta SPAD-502 e M4: testemunha sem a aplicação de $N$. O delineamento experimental foi o inteiramente casualizado com quatro tratamentos e cinco repetições. A produtividade de grãos no tratamento baseado nas leituras do clorofilômetro portátil (M3) foi semelhante a do tratamento normalmente recomendado para o feijoeiro irrigado (M2), porém, foram aplicados $60 \mathrm{~kg} \mathrm{ha}^{-1}$ de $N$ a menos. Os níveis críticos mínimos de índice relativo de clorofila para obtenção de produtividade superior a $90 \%$ da máxima foram, respectivamente, de 43 e 46 aos 28 e 49 dias após emergência do feijoeiro.

Palavras-chave: Phaseolus vulgaris, Manejo de nitrogênio, clorofilômetro, clorofila, índice relativo de clorofila, índice de suficiência de nitrogênio.

\section{ABSTRACT}

The research showed that readings of transmittance obtained with portable chlorophyll meter can indicate timing of higher demand for nitrogen by plants, if there is need of $N$ topdressing. This was the objective of this study. Were the following $N$ management practices were evaluated for irrigated commum bean: M1: application of $240 \mathrm{~kg} \mathrm{~N} \mathrm{ha-1,} 80 \mathrm{~kg}$ at sowing, $80 \mathrm{~kg} 15$ days after plant emergence and $80 \mathrm{~kg} 30$ days after plant emergence. M2: applying $120 \mathrm{~kg} N \mathrm{ha}^{-1}(30 \mathrm{~kg} \mathrm{~N}$ at sowing $+45 \mathrm{~kg} N 15$ days after emergence $+45 \mathrm{~kg} \mathrm{~N} 30$ days after emergence); M3: Application of $30 \mathrm{~kg} \mathrm{~N} \mathrm{ha}^{-1}$ at sowing and $N$ topdressing based on use of chlorophyll meter Minolta SPAD-502, and M4: control (without N). Experimental design was randomized complete plots with four treatments and five replications. Grain yield in the treatment based on the readings of portable chlorophyll meter (M3) was similar to treatment generally recommended to growers for irrigated dry bean (M2), however $60 \mathrm{~kg} \mathrm{~N} \mathrm{ha}^{-1}$ less was applied. Minimum critical levels of chlorophyll relative index to obtain $90 \%$ maximum grain yield of dry bean were 43 and 46 at 28 and 49 days after emergence of commum bean., respectively.

Key words: Nitrogen management, Phaseolus vulgaris, chlorophyll meter, chlorophyll, chlorophyll relative index, nitrogen sufficient index.

\section{INTRODUÇÃO}

$\mathrm{O}$ aparecimento de deficiência de $\mathrm{N}$ em plantas é muito comum em quase todos os solos, sendo caracterizada por um amarelecimento generalizado das folhas, que se inicia pelas mais velhas (CARVALHO et al., 2003). Ainda não existe um método laboratorial que permita avaliar satisfatoriamente a capacidade do solo em fornecer $\mathrm{N}$ às plantas, dada à complexidade e às interações entre os processos de transformação do nutriente no solo e as condições climáticas, dificultando assim prever a necessidade de adubação nitrogenada para uma dada cultura. Desse modo, as recomendações de adubação nitrogenada são, geralmente, baseadas

IEmbrapa Arroz e Feijão, CP 179, 75375-000, Santo Antônio de Goiás, GO, Brasil. E-mail: morel@cnpaf.embrapa.br. *Autor para correspondência.

"Universidade Federal de Lavras (UFLA), Lavras, MG, Brasil. 
em curvas de resposta da planta ao nitrogênio, obtidas em ensaios de campo, em que os dados de produtividade de grãos, absorção de $\mathrm{N}$ ou acúmulo de matéria seca pela planta são ajustados a equações matemáticas, que expressam a resposta da planta ao nutriente.

No feijoeiro, os nutrientes são absorvidos durante todo seu ciclo, todavia, a maior demanda para a produção de grãos ocorre até a fase que antecede a floração, cerca de 45 dias após a emergência das plântulas (BARBOSA FILHO \& SILVA, 2000), dependendo do teor de matéria orgânica do solo, do sistema de plantio e dos resíduos vegetais presentes na superfície do solo. A recomendação de adubação para o feijoeiro mais rotineiramente utilizada tem sido a aplicação de parte do nitrogênio no sulco, junto ao fósforo e ao potássio por ocasião da semeadura, e parte em cobertura (BARBOSA FILHO \& SILVA, 1994; BARBOSAFILHO \& SILVA, 2000; BARBOSAFILHO et al., 2004, 2005). Nesses trabalhos, as doses e as épocas de aplicação do $\mathrm{N}$ em cobertura foram prédefinidas, sem considerar as necessidades e o momento de maior demanda do nutriente pela cultura. A conseqüência disso pode ser a sub ou superestimativa da dose de $\mathrm{N}$ utilizada, acarretando, por um lado, queda de rendimento de grãos e, por outro, risco de poluição ambiental e diminuição de lucro do agricultor pelo uso desnecessário de fertilizantes (ARGENTA et al., 2003).

No Brasil, a eficiência de uso dos fertilizantes nitrogenados na produção das culturas ainda permanece muito baixa e uma das explicações para esse fato está relacionada com a falta de sincronismo entre a época de aplicação $\mathrm{N}$ e a época de maior demanda da planta. Visando melhorar este sincronismo, alguns autores têm sugerido o monitoramento do teor de $\mathrm{N}$ da folha e de clorofila, por meio do uso de clorofilômetro portátil, que se correlacionam positivamente entre si e com a produtividade de grãos (PENG et al., 1993; FURLANI JÚNIOR et al., 1996; STONE et al., 2002; HUSSAIN et al., 2000; ARGENTA et al., 2003; CARVALHO et al., 2003). O princípio de funcionamento do aparelho utilizado nos estudos (modelo Minolta SPAD-502) é simples e baseia-se na quantidade de luz de comprimentos de onda variando de $650 \mathrm{~nm}$ a $940 \mathrm{~nm}$ que atravessa a folha (transmitância), medindo indiretamente a concentração relativa de clorofila e expressa como Índice Relativo de Clorofila (IRC) (GUIMARÃES et al., 1998; NUNES et al., 2003).

A predição da necessidade de aplicação de $\mathrm{N}$ em cobertura para arroz irrigado pode ser determinada utilizando-se o Índice de Suficiência de Nitrogênio (ISN), que é calculado a partir das leituras do clorofilômetro em relação a parcelas de referência, bem fertilizadas, e ainda sugere que a dose de $\mathrm{N}$ destas parcelas deve representar, no mínimo, o dobro da dose recomendada de modo a garantir o não aparecimento de deficiência de N (HUSSAIN et al., 2000). De acordo com os autores, a aplicação de $\mathrm{N}$ em cobertura deve ser efetuada todas as vezes que o ISN for inferior a $90 \%$ do tratamento de referência.

Os objetivos deste trabalho foram predizer a necessidade de suplementação de $\mathrm{N}$ em cobertura para o feijoeiro irrigado, a partir do índice relativo de clorofila nas folhas medidos com clorofilômetro, e avaliar se o Índice de Suficiência de Nitrogênio (ISN) pode servir de indicativo de resposta do feijoeiro irrigado à aplicação de $\mathrm{N}$.

\section{MATERIAL E MÉTODOS}

O experimento foi realizado de julho a setembro de 2004 na Fazenda Guaíba, localizada no município de Unaí, Minas Gerais (MG). O solo do local é caracterizado como um Latossolo vermelho distroférrico. Utilizou-se o sistema plantio direto em área coberta com palhada de milho e a cultivar de feijão "Pérola”, semeada no espaçamento entrelinhas de 0,45 m e aproximadamente 12 sementes viáveis por metro de sulco. As parcelas mediam $10 \mathrm{~m}$ x $8 \mathrm{~m}$, sendo considerada como área útil $9 \mathrm{~m}^{2}$. Antes da semeadura foi tomada uma amostra de solo que foi analisada e os resultados revelaram: $\mathrm{pH}\left(\mathrm{H}_{2} \mathrm{O}\right), 5,8$; $\mathrm{P}$ (Mehlich 1), 3,0 $\mathrm{mg} \mathrm{dm}^{-3}$; K trocável (Mehlich 1), 61 $\mathrm{mg} \mathrm{dm}^{-3}$; Ca + Mg trocáveis (em $\mathrm{KCl} \mathrm{0,1} \mathrm{N),27,9mmol} \mathrm{cm}{ }^{-3} ; \mathrm{Al}^{+3}$, $1,0 \mathrm{mmol} \mathrm{cm}^{-3}$; CTC a pH 7,0, 78,9 $\mathrm{mmol} \mathrm{cm}^{-3}$; sendo o $\mathrm{H}$ + Al extraídos em acetato de cálcio tamponado a $\mathrm{pH}$ 7,0, V, 35,4\% e matéria orgânica, 22 g kg-1 determinada pelo método de Walkley e Black.

A adubação básica de fósforo e potássio foi a usual nas propriedades locais para a cultura do feijoeiro e consistiu de $100 \mathrm{~kg} \mathrm{ha}^{-1}$ de $\mathrm{P}_{2} \mathrm{O}_{5}$ e $70 \mathrm{~kg} \mathrm{ha}^{-1}$ de $\mathrm{K}_{2} \mathrm{O}$ nas formas de superfosfato triplo e cloreto de potássio, respectivamente. O delineamento experimental foi o inteiramente casualizado com quatro tratamentos, constituídos por manejos do N, e cinco repetições. Os manejos do $\mathrm{N}$ avaliados foram os seguintes: M1: Aplicação de $240 \mathrm{~kg} \mathrm{ha}^{-1}$ de $\mathrm{N}$, sendo 80kg na semeadura, $80 \mathrm{~kg}$ aos 15 dias após emergência (dae) e $80 \mathrm{~kg}$ aos 30 dae, sendo este denominado de tratamento de referência (garantia de não ocorrência de deficiência de N), M2: Aplicação de N baseada na recomendação local recomendada por BARBOSA FILHO et al. (2005) para a cultura do feijoeiro irrigado, ou seja, $30 \mathrm{~kg} \mathrm{ha}^{-1}$ de $\mathrm{N}$ na semeadura $+45 \mathrm{~kg}$ aos 15 dae + 45kg aos 30 dae; M3: Aplicação de $30 \mathrm{~kg} \mathrm{ha}^{-1}$ de N no plantio e aplicação em cobertura baseada no uso do clorofilômetro Minolta SPAD-502, usando o índice de 
suficiência de $\mathrm{N}<90 \%$ proposto por HUSSAIN et al. (2000). Com base neste princípio, foram aplicados no M3 mais $30 \mathrm{~kg} \mathrm{ha}^{-1}$ de $\mathrm{N}$ aos 28 dae, perfazendo o total de $60 \mathrm{~kg} \mathrm{ha}^{-1}$ de N; e M4: testemunha sem $\mathrm{N}$.

As leituras no clorofilômetro iniciaram-se aos 28 dae. A partir desta data, as leituras eram tomadas, semanalmente, em três pontos da parcela, sendo que em cada ponto as leituras foram tomadas de seis plantas, sendo feitas cinco leituras/folha (penúltima folha trifoliada completamente desenvolvida), em todo o limbo da folha, exceto nervuras, somando, assim, 30 leituras, totalizando, portanto, 90 leituras/parcela. Os valores de densidade ótica (IRC) foram usados para determinar o teor total de clorofila pela equação linear $y=-0,152+0,0996 x$, determinada por BARNES et al. (1992). Na análise de $\mathrm{N}$, foram utilizadas as mesmas folhas em que foi realizada a leitura do IRC. As folhas foram coletadas semanalmente, no momento da medição do IRC e acondicionadas em sacos de papel e secas em estufa até peso constante. Depois de pesadas, foram moídas e submetidas à digestão sulfúrica para a determinação do teor de N, conforme MORAIS \& RABELO (1986). O acúmulo de $\mathrm{N}$ nas folhas foi calculado pela multiplicação da massa seca das folhas pelo teor correspondente de $\mathrm{N}$ no material vegetal e os dados ajustados por meio de análise de regressão. A produtividade de grãos foi determinada em área útil de $27 \mathrm{~m}^{2}$ da parcela, sendo a umidade dos grãos ajustada a $130 \mathrm{~g} \mathrm{~kg}^{-1}$. A estimativa da eficiência agronômica foi feita com base nas produtividades de grãos obtidas nas parcelas com e sem a aplicação de N (Eficiência agronômica $=\Delta \mathrm{kg}$ de grãos $/ \Delta \mathrm{kg}$ de $\mathrm{N}$ aplicado). Os dados foram submetidos à análise de variância e a comparação de médias entre os tratamentos foi efetuada pelo teste de Tukey $(\mathrm{P} \leq 0,05)$.
O critério de Cate-Nelson de determinação de níveis críticos foi adotado plotando-se no eixo da abscissa (x) os valores de IRC e na coordenada (y) as produtividades relativas (\%), calculadas em relação à maior produtividade de grãos, em duas épocas de crescimento quando geralmente ocorre a deficiência de $\mathrm{N}$ na cultura do feijoeiro, uma aos 28 e outra aos 49 dias após emergência em pleno florescimento das plantas. Os valores críticos de IRC foram determinados nos níveis de $90 \%$ de produtividade máxima (CATE \& NELSON, 1971).

\section{RESULTADOS E DISCUSSÃO}

Os resultados da tabela 1 mostram que o efeito da aplicação de $\mathrm{N}$ foi positivo sobre a produtividade de grãos em relação à testemunha sem N (M4). A produtividade de grãos no tratamento baseado no ISN $<90 \%$, como indicativo da necessidade de adubação nitrogenada em cobertura (M3), foi semelhante a do tratamento de recomendação local aos produtores no cultivo do feijoeiro irrigado (M2). A partir desse índice, a adubação nitrogenada em cobertura consumiu $60 \mathrm{~kg} \mathrm{ha}^{-1}$ a menos, em comparação com a recomendação local, baseada em épocas pré-fixadas para a aplicação de $\mathrm{N}$ em cobertura (M2). Com isso, no M3, a eficiência agronômica (EA) foi maior do que no tratamento baseado na recomendação local (Tabela 1). A EA, por definição, é maior quanto menor for a quantidade de $\mathrm{N}$ aplicada. Portanto, o fato de a EA ser maior no tratamento com menos $\mathrm{N}$ aplicado somente passa a ser importante se, comparado com o tratamento usual, não houver redução de produtividade de grãos, fato este observado no presente trabalho (Tabela 1). Nesse sentido, pode-se considerar que a estratégia de

Tabela 1 - Produtividade de grãos, acúmulo de $\mathrm{N}$ nas folhas na fase de florescimento pleno e eficiência agronômica pelo feijoeiro irrigado, cultivado em sistema plantio direto com diferentes manejos de adubação nitrogenada em cobertura no município de Unaí, MG, 2004 .

\begin{tabular}{|c|c|c|c|}
\hline Manejo & $\begin{array}{l}\text { Produtividade de grãos } \\
\qquad \mathrm{kg} \mathrm{ha}^{-1}\end{array}$ & $\begin{array}{l}\text { Acúmulo de } \mathrm{N} \text { nas folhas }{ }^{(1)} \\
\qquad \mathrm{mg} \mathrm{g}^{-1}\end{array}$ & $\begin{array}{c}\text { Eficiência agronômica } \\
\text { kg grãos } \text { kg }^{-1} \text { de } \mathrm{N}\end{array}$ \\
\hline M1 $\left(240^{(2)}\right.$ & $3.251 \mathrm{a}^{(3)}$ & $31,76 a$ & 5,7 \\
\hline M2 (120) & $2.522 b$ & $24,88 a b$ & 5,4 \\
\hline M3 (60) & $2.527 b$ & $21,16 \mathrm{bc}$ & 10,9 \\
\hline M4 (0) & $1.875 c$ & $13,98 c$ & - \\
\hline CV (\%) & 9,4 & 18,70 & - \\
\hline Valor F & $29,217 * *$ & $14,883^{*}$ & - \\
\hline
\end{tabular}

*Significativo a $\mathrm{P}<0,05$. **Significativo a $\mathrm{P}<0,01$. M1 $=80 \mathrm{~kg} \mathrm{ha}^{-1}$ na semeadura, $80 \mathrm{~kg} \mathrm{ha}^{-1}$ aos 15 dae e $80 \mathrm{~kg}^{-1}$ aos 30 dae. M2 = Recomendação local $=30 \mathrm{~kg} \mathrm{ha}^{-1}$ na semeadura, $45 \mathrm{~kg} \mathrm{ha}^{-1}$ aos 15 dae e $45 \mathrm{~kg} \mathrm{ha}^{-1}$ aos 30 dae. M3 = 30kg ha ${ }^{-1}$ de $\mathrm{N}$ foram aplicados na semeadura e $30 \mathrm{~kg}$ foram aplicados aos 28 dias após a emergência, quando as leituras do clorofilômetro indicaram ISN <90\%, totalizando $60 \mathrm{~kg} \mathrm{ha}{ }^{-1}$. M4 = Testemunha sem nitrogênio. ${ }^{(1)}$ Consideraram-se as folhas coletadas aos 49 dias após a emergência das plantas em pleno florescimento do feijoeiro; ${ }^{(2)}$ Representam as quantidades totais de $\mathrm{N}$ aplicadas durante o ciclo de crescimento do feijoeiro irrigado. ${ }^{(3)} \mathrm{Na}$ coluna, médias seguidas pela mesma letra não diferem pelo teste Tukey a 5\% de probabilidade de erro. 
se usar o clorofilômetro portátil (Monolta SPAD 502), tendo como indicador o ISN $<90 \%$, foi adequada para determinar o momento para a aplicação de N, já que as produtividades de grãos foram semelhantes nos dois tratamentos. Evidencia-se, com isso, que a adubação nitrogenada de cobertura baseada em épocas préfixadas pode não ser a mais adequada para as condições de cultivo em que foi realizado o presente trabalho.

Os índices relativos de clorofila foram crescentes com o tempo, estabilizando-se a partir do pleno florescimento da cultura (49 dae) (Figura 1a). Isso indica que após esta fase de crescimento do feijoeiro a resposta da cultura ao $\mathrm{N}$ é pequena, em razão da menor demanda, e que o teor de clorofila nas folhas se estabiliza, tornando-se ineficaz o uso do aparelho a partir desta fase, como indicador da necessidade da aplicação de $\mathrm{N}$ em cobertura. Na figura 1, é feita a comparação entre as duas estratégias para verificação da necessidade de aplicação do $\mathrm{N}$, uma baseada no IRC e outra, no ISN $<90 \%$. Aos 28 dae, foram observados valores de IRC $<90 \%$ do índice de suficiência no tratamento baseado no clorofilômetro, indicando a necessidade da aplicação de $\mathrm{N}$, época em que foram aplicados $30 \mathrm{~kg} \mathrm{ha}^{-1}$ de $\mathrm{N}$.

Na fase inicial de crescimento do feijoeiro, observaram-se sintomas visuais de deficiência de $\mathrm{N}$ no tratamento sem aplicação de N (M4); porém, a partir dos 42 dae as plantas adquiriram a tonalidade verde, resultando em valores de índices relativos de clorofila muito próximos das leituras observadas nas parcelas adubadas com N. A explicação se deve ao processo de mineralização do $\mathrm{N}$ dos resíduos vegetais deixados na superfície do solo pelo cultivo anterior de milho.

$\mathrm{O}$ fato de as plantas tornarem-se verdes com o tempo de mineralização do $\mathrm{N}$ contido nos resíduos vegetais é indicativo de que os IRC podem ser afetados, em função da quantidade e da qualidade dos resíduos vegetais antecedentes. Portanto, dependendo da época em que os IRC são medidos, podem ocorrer erros de interpretação, uma vez que podem não indicar deficiência do nutriente ocorrida na fase inicial de crescimento da planta, como foi constatado e comprovado a partir dados de produtividade de grãos apresentados na tabela 1. Tomada esta precaução, pode-se considerar que o clorofilômetro Minolta SPAD502 é promissor como instrumento indicativo da época em que se deve iniciar a aplicação de $\mathrm{N}$ em cobertura no feijoeiro irrigado. A adubação em cobertura baseada neste critério resulta em maior EA do $\mathrm{N}$ do que a aplicação de $\mathrm{N}$ baseada na recomendação local.

Os valores de IRC e os teores de clorofila e o acúmulo de $\mathrm{N}$ nas folhas foram altamente relacionados (Figuras 2a,b), possibilitando assim avaliar a necessidade de aplicação de $\mathrm{N}$ em cobertura no feijoeiro irrigado, o que confirma resultados de outros autores (BARNES et al., 1992; PENG et al., 1993; CARVALHO et al., 2003; FURLANI JÚNIOR et al., 1996; STONE et al., 2002).

Os teores de clorofila e os valores de IRC aumentaram à medida que aumentaram o acúmulo de $\mathrm{N}$ nas folhas. A utilização desses valores tem sido uma estratégia simples e eficiente de manejo do $\mathrm{N}$ em várias

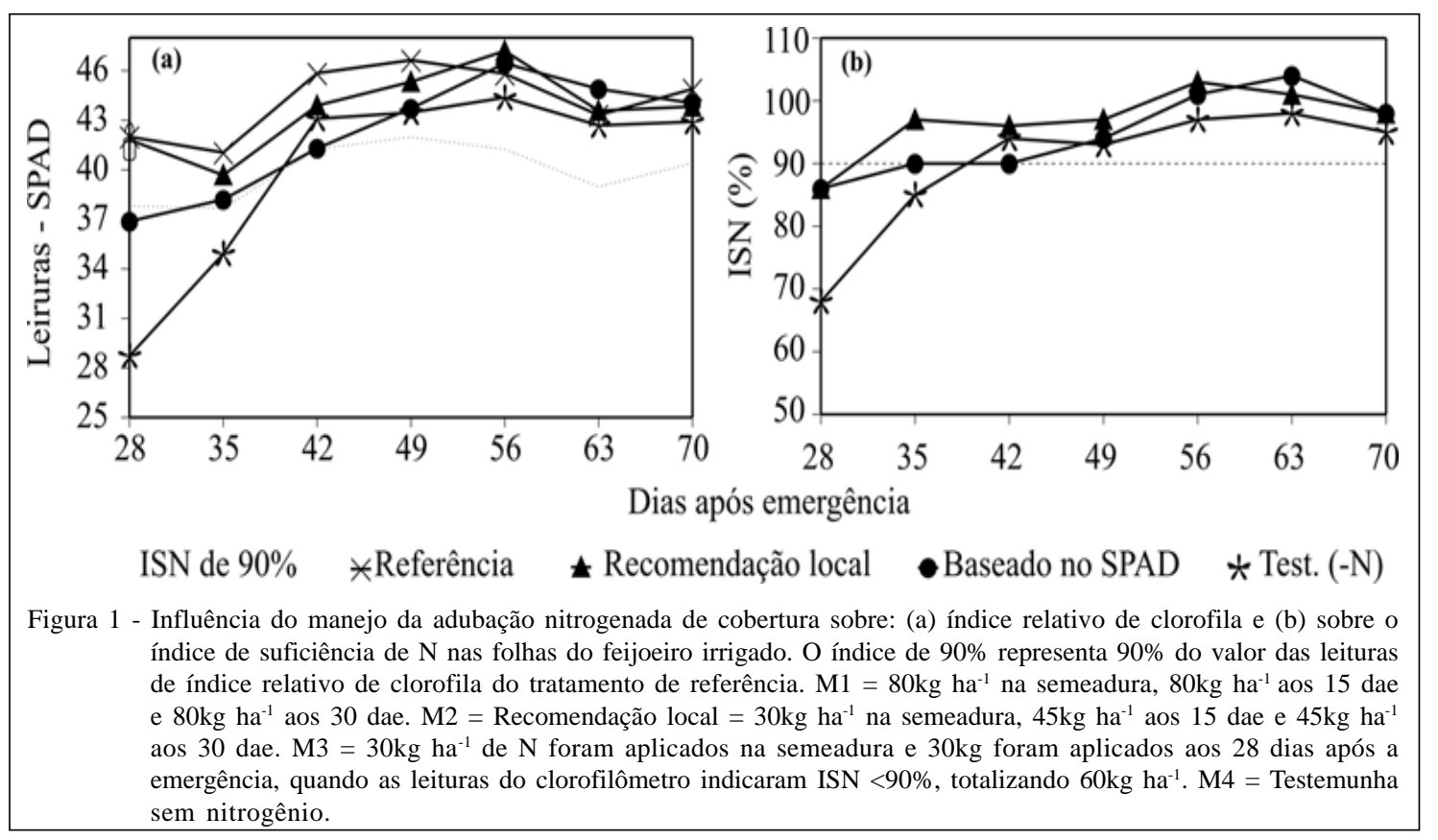

Ciência Rural, v.38, n.7, out, 2008. 

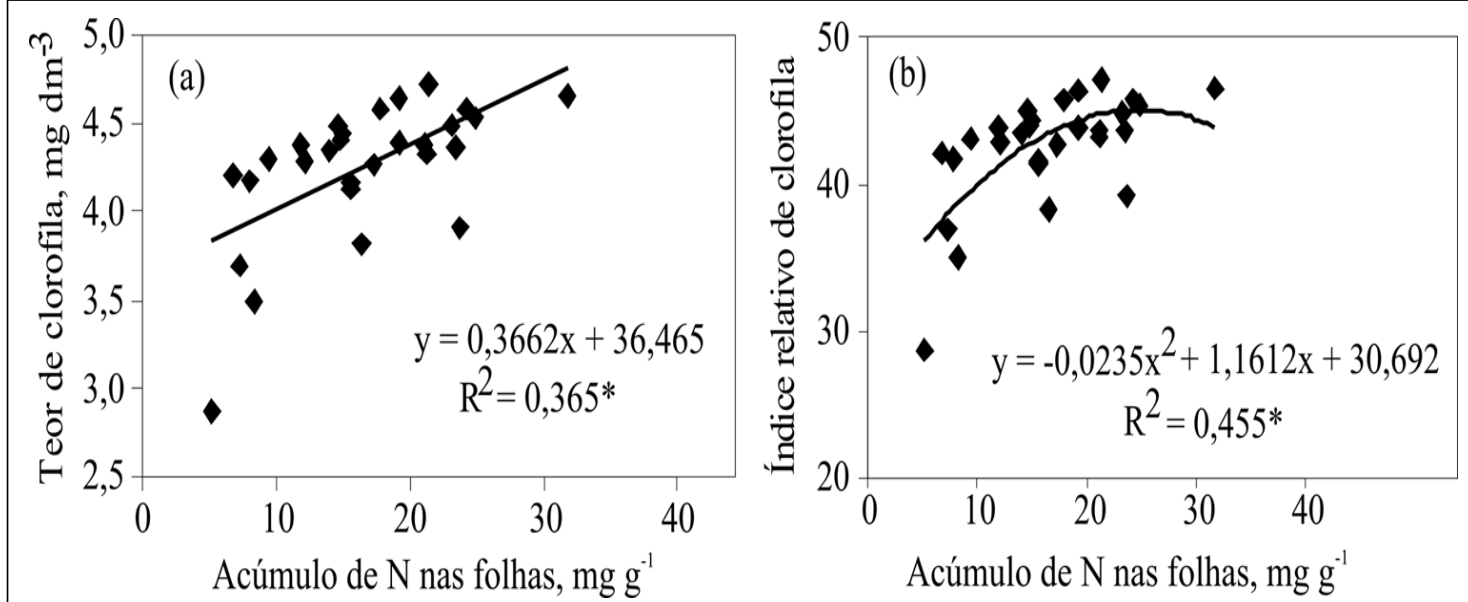

Figura 2 - Relação entre teor de clorofila e acúmulo de $\mathrm{N}$ nas folhas (a) e entre índice relativo de clorofila e acúmulo de $\mathrm{N}$ nas folhas (b) do feijoeiro irrigado cultivado no sistema plantio direto, sob diferentes manejos de adubação nitrogenada de cobertura. *significativo a $5 \%$.

culturas, tais como milho (CHAPMAN \& BARRETO, 1997), arroz irrigado (HUSSAIN et al., 2000) e feijão (CARVALHO et al., 2003; STONE et al., 2002; FURLANI JÚNIOR et al., 1996). Porém, ressalta-se que o valor crítico de IRC, ou seja, o nível abaixo do qual espera-se uma resposta ao $\mathrm{N}$ pode variar com a espécie, a cultivar e as condições climáticas, especialmente radiação solar, fornecimento de $\mathrm{N}$ e práticas de manejo (SHUKLA et al., 1986).

Aplicando-se o método de CATE \& NELSON (1971) para a determinação do nível crítico aos dados de produtividade de grãos, foi estabelecido o nível crítico de IRC de 43 unidades-SPAD aos 28 dae e de 46 aos 49 dae, em pleno florescimento das plantas. Isso indica que, nas condições experimentais, valores acima desses referenciais não indicam a necessidade de suplementação de N (Figura 3a, b) e que a relação entre IRC e teor de $\mathrm{N}$ nas folhas depende do estádio de crescimento da planta. Este resultado é semelhante ao obtido por STONE et al. (2002). Segundo CATE \& NELSON (1971), para uma técnica em estudo ser considerada adequada, todos os pontos deveriam estar localizados dentro dos quadrantes inferior esquerdo e superior direito e aqueles que se encontram nos quadrantes inferior direito e superior esquerdo indicam quando o $\mathrm{N}$ suplementar de cobertura não deve ser recomendado (Figura 3a, b). Portanto, aos 28 dae, os valores de IRC abaixo do nível crítico de 43 correspondentes a valores de produtividades situados no quadrante superior esquerdo indicam alta probabilidade de resposta à aplicação de $\mathrm{N}$ em cobertura.
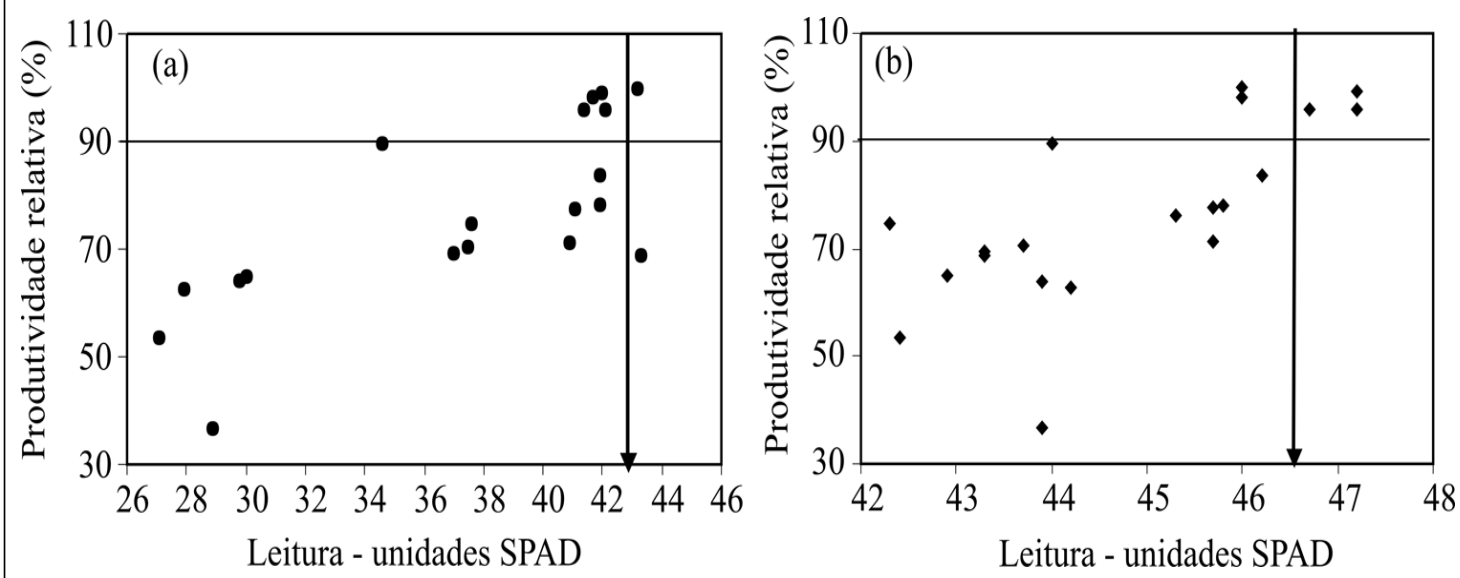

Figura 3 - Relação entre índice relativo de clorofila e porcentagem da produtividade máxima de grãos e nível crítico de leitura de índice relativo de clorofila (a) antes da aplicação de $\mathrm{N}$ em cobertura (aos 28 dae) e (b) no florescimento pleno (aos 49 dae), segundo CATE \& NELSON (1971). 


\section{CONCLUSÕES}

O clorofilômetro Minolta SPAD-502 indica a época em que se deve iniciar a aplicação de $\mathrm{N}$ em cobertura no feijoeiro irrigado. Os níveis críticos mínimos de índice relativo de clorofila para obtenção de produtividade superior a $90 \%$ da máxima foram, respectivamente, de 43 e 46 aos 28 e 49 dias após emergência do feijoeiro.

O Índice de Suficiência de Nitrogênio menor que $90 \%$ constitui uma técnica simples e eficiente de manejo do $\mathrm{N}$, permitindo diagnosticar o nível de $\mathrm{N}$ na folha e determinar a melhor época para a aplicação de $\mathrm{N}$, melhorando a eficiência de uso do $\mathrm{N}$ pelo feijoeiro.

\section{AGRADECIMENTOS}

Ao Engenheiro Agrônomo Luís Joaquim Missio, administrador da fazenda Guaíba, pela concessão da área experimental e pelo apoio na condução do experimento.

\section{REFERÊNCIAS}

ARGENTA, G. Adubação nitrogenada em milho pelo monitoramento do nível de nitrogênio na planta por meio do clorofilômetro. Revista Brasileira de Ciência do Solo, Viçosa, v.27, n.1, p.109-119, 2003.

BARBOSA FILHO, M.P. Fontes, doses e parcelamento da adubação nitrogenada em cobertura para feijoeiro comum irrigado. Ciência e Agrotecnologia, Lavras, v.29, n.1, p.6976, 2005.

BARBOSA FILHO, M.P. Fontes e métodos de aplicação de nitrogênio em feijoeiro irrigado submetido a três níveis de acidez do solo. Ciência e Agrotecnologia, Lavras, v.28, n.4, p.785792, 2004.

BARBOSA FILHO, M.P.; SILVA, O.F. da. Adubação e calagem para o feijoeiro irrigado em solo de cerrado. Pesquisa Agropecuária Brasileira, Brasília, v.35, n.7, p.1317-1324, 2000.

BARBOSA FILHO, M.P.; SILVA, O.F. da. Aspectos agroeconômicos da calagem e da adubação nas culturas de arroz e feijão irrigados por aspersão. Pesquisa Agropecuária Brasileira, Brasília, v.29, n.11, p.1657-1667, 1994.
BARNES, J.D. et al. A reappraisal of the use of DMSO for the extraction and determination of chlorophylls a and b in lichens and higher plants. Environmental and Experimental Botany, Oxford, v.32, p.85-100, 1992.

CARVALHO, M.A.C. et al. Doses e épocas de aplicação de nitrogênio e teores foliares deste nutriente e de clorofila em feijoeiro. Revista Brasileira de Ciência do Solo, Viçosa, v.27, n.3, p.445-450, 2003.

CATE, R.B.; NELSON, L.A. A simple statistical procedure for partitioning soil correlation data into classes. Soil Science Society of America Proceedings, Madison, v.35, p.658-660, 1971.

CHAPMAN, S.C.; BARRETO, H.J. Using a chlorophyll meter to estimate specific leaf nitrogen of tropical maize during vegetative growth. Agronomy Journal, v.89, p.557-562, 1997

FURLANI JÚNIOR, E. et al. Correlação entre leituras de clorofila e níveis de nitrogênio aplicados em feijoeiro. Bragantia, Campinas, v.55, n.1, p.171-175, 1996.

GUIMARÃES, T.G. . Determinação dos teores de nitrogênio na seiva do tomateiro por meio de medidor portátil. Horticultura Brasileira, Brasília, v.16, p.144-151, 1998.

HUSSAIN, F. et al. Use of chlorophyll meter sufficiency indices for nitrogen management of irrigated rice in Asia. Agronomy Journal, Madison, v.92. n.5, p.875-879, 2000.

MORAIS, J.F.V.; RABELO, N.A. Um método simples para a digestão de amostras de plantas. Goiânia: EMBRAPACNPAF, 1986. 10p. (EMBRAPA-CNPAF Documentos, 12).

NUNES, J.C.S. et al. Efeito da palhada de sorgo localizada na superfície do solo em característica de plantas de soja e milho. Revista Ceres, Viçosa, v.50, n.287, p.115-126, 2003.

PENG, S. Adjustment for specific leaf weight improves chlorophyll meter estimate of rice leaf nitrogen concentration. Agronomy Journal, Madison, v.85, n.5, p.987-990, 1993.

SHUKLA, A.K. et al. Calibrating of leaf color chart for nitrogen management in different genotypes of rice and wheat in a system perspective. Agronomy Journal, Madison, v.96, p.1606-1621, 2004.

STONE, L.F. et al. Uso do clorofilômetro SPAD-502 na estimativa do nitrogênio foliar específico e da produtividade do feijoeiro. In: CONGRESSO NACIONAL DE PESQUISA DE FEIJÃO, 7., 2002, Viçosa. Resumos expandidos... Viçosa: UFV, 2002. p.743-746. 